\title{
Maren Gottschalk, Johannes Gutenberg. Mann des Jahrtausends
}

\section{Charlotte Kempf}

\section{OpenEdition}

\section{Journals}

Édition électronique

URL : http://journals.openedition.org/ifha/9710

DOI : 10.4000/ifha.9710

ISSN : 2198-8943

\section{Éditeur}

IFRA - Institut franco-allemand (sciences historiques et sociales)

Référence électronique

Charlotte Kempf, " Maren Gottschalk, Johannes Gutenberg. Mann des Jahrtausends », Revue de l'IFHA [En ligne], Date de recension, mis en ligne le 12 novembre 2018, consulté le 24 septembre 2020. URL http://journals.openedition.org/ifha/9710 ; DOI : https://doi.org/10.4000/ifha.9710

Ce document a été généré automatiquement le 24 septembre 2020

(CIFHA 


\title{
Maren Gottschalk, Johannes Gutenberg. Mann des Jahrtausends
}

\author{
Charlotte Kempf
}

Plusieurs travaux académiques ont déjà été consacrés à la vie et à l'œuvre de Johannes Gutenberg : Gutenberg - Leben und Werk. Eine Einführung de Hans-Joachim Koppitz, la monographie de Wolfgang von Stromer Gutenbergs Geheimnis. Von Turfan zum Karlstein die Seidenstraße als Mittler der Druckverfahren von Zentralasien nach Mitteleuropa ou encore les publications de Stephan Füssel Johannes Gutenberg et Gutenberg und seine Wirkung, pour n'en citer que les plus notables. Des ouvrages de vulgarisation se sont également emparé du sujet : ainsi, en 1993, un livre de 368 pages écrit par Andreas Venzke fut publié sous le titre de Johannes Gutenberg. Der Erfinder des Buchdrucks; plus récemment, en 2016, Klaus-Rüdiger Mai a consacré un épais volume au père de l'imprimerie avec son Gutenberg. Der Mann, der die Welt veränderte, également destiné aux néophytes. À l'occasion de la commémoration du $550^{\mathrm{e}}$ anniversaire de sa disparition paraît aujourd'hui une nouvelle biographie, dans la tradition de ces ouvrages grand public ; l'auteur en est Maren Gottschalk, le titre Johannes Gutenberg. Mann des Jahrtausends. L'ouvrage n'a pas de prétentions scientifiques et décevra celui qui y chercherait les derniers apports de la recherche au dossier Guttenberg. M. Gottschalk s'en tient à l'essentiel, et adopte pour cela un ton plaisamment décontracté qui sied au caractère divertissant de l'ouvrage et ravira certainement le néophyte intéressé par le sujet. On n'assomme certes pas le lecteur de notes de bas pages, pas plus qu'on ne s'embarque dans de longues digressions. Pendant 160 pages, Gottschalk s'applique à tracer les grandes lignes de la biographie de Gutenberg en y mêlant des développements sur l'histoire de la ville de Mayence, thèmes déjà traités par la littérature spécialisée. Il convient de noter l'élégance de la mise en page ainsi que la réussite générale de la conception graphique de cet ouvrage au format maniable, agréable à tenir en main et riche de nombreuses illustrations - même si concernant ces dernières, le lecteur habitué aux informations historiquement plus précises aurait parfois souhaité des références plus exactes que l'indication de Wikimedia commons. Gottschalk peint Gutenberg en homme d'action qui, en bon vivant, sut profiter des plaisirs de ce monde, 
fut un homme d'affaires avisé et opiniâtre et tira le meilleur de ses succès commerciaux et de ses échecs.

Selon M. Gottschalk, Gutenberg eut toujours à cœur de garder le secret et de maximiser le profit de son invention, c'est pourquoi il dut tout de même s'associer avec des experts de son époque pour se faire expliquer certains procédés techniques. Tous ses travaux auraient tendu à un but concret, celui de produire grâce l'imprimerie des livres plus rapidement et de manière moins coûteuse, mais sans sacrifier à la qualité. Cette vision peut être considérée comme simpliste, et l'auteur a tendance à exagérer cette image, mais pour un public ignorant du sujet, l'ouvrage a le mérite de faire de Gutenberg un personnage plus saisissable. M. Gottschalk se permet de remplir certaines lacunes qui, à ce jour, existent encore dans sa biographie ; par exemple quant à la question de savoir où Gutenberg séjourna entre 1428 et 1434, elle avance l'hypothèse qu'il aurait pu se trouver à Bâle ou à Nuremberg. Mais la source historique qui nous livrera l'information permettant d'éclaircir ce mystère n'a pas encore été découverte, de sorte que cette hypothèse n'apporte aucune révélation, et n'offre pas non plus, à vrai dire, de nouvelles pistes à la réflexion. Elle a tout de même le mérite de permettre au lecteur de réaliser que certaines zones d'ombres continuent d'entourer cette période de la vie de Gutenberg ; en effet, notre homme vivait peut-être alors dans une tout autre ville. Bien que l'ouvrage de M. Gottschalk ne soit pas exempt de petites erreurs, il permet de saisir avec clarté les grands moments de la vie de Gutenberg, et offre une perspective sur l'histoire européenne de l'imprimerie qui continue à se développer au cours du XV $\mathrm{XV}^{\mathrm{e}}$ siècle. M. Gottschalk avait déjà rédigé des ouvrages biographiques de vulgarisation, entre autres sur Nelson Mandela, Andy Warhol et Frida Kahlo, où elle faisait également preuve d'un véritable talent à s'emparer d'un sujet historique pour un public non averti ; on sent cette expérience à la lecture de son dernier ouvrage. Ce livre plaisant et facile à lire ne peut certes pas prétendre à remplacer les biographies scientifiques déjà parues, mais il a le mérite de contribuer à rendre la vie et l'œuvre de ce personnage essentiel accessibles au grand public.

\section{INDEX}

Index chronologique : Moyen Âge, Période moderne

Thèmes : Histoire de la culture

\section{AUTEURS}

\section{CHARLOTTE KEMPF}

Ruprecht-Karls-Universität, Heidelberg 